\title{
EMT and Anti-EMT Strategies in Cancer
}

\author{
M. A. Nezami ${ }^{1}$, Steven Hager ${ }^{2}$, Jessica Garner ${ }^{1}$ \\ ${ }^{1}$ Pacific Medical Center of Hope, Fresno, CA, USA \\ ${ }^{2}$ Ccare, Fresno, CA, USA \\ Email: amnezami@yahoo.com
}

Received 23 September 2015; accepted 25 October 2015; published 29 October 2015

Copyright (C) 2015 by authors and Scientific Research Publishing Inc.

This work is licensed under the Creative Commons Attribution International License (CC BY).

http://creativecommons.org/licenses/by/4.0/

(c) (7) Open Access

\begin{abstract}
This article discusses the role of epithelial mesenchymal transition (EMT) and addresses the scientific merits on epigenetic regulation of EMT. The importance of EMT as a prognostic biomarker is explored and the rationale on application of multitargeted epigenetic therapy is discussed. We describe a literature review of the epigenetic influence of such process and we present a potentially effective method to reverse the epigenetic switch in favor for MET, in a clinical setting. A case series of advanced solid tumors are summarized aiming at generating hypothesis for the future recommendations for clinical trials targeting the tumor's biological behavior through inhibition of EMT. Hypothesis: We propose an integral and integrative approach that can modify tumor's biological behavior through inhibition of EMT, and further reduce the chances of metastasis, that can translate to improved outcome and patient's survival in advanced disease.
\end{abstract}

\section{Keywords}

Cancer, EMT, Anti-EMT, Epigenetic, CTC, ctDNA

\section{Introduction}

Therapeutically, "generating” stem cells during the process of EMT has recently been described as a tool for treating diabetes mellitus type I [1]. Logically, the reverse, "inhibition of EMT” or induction of a phenomenon known as "MET" (mesenchymal epithelial transition) should pertain to the treatment of cancer. Epithelial mesenchymal transition (EMT) entails significant morphological and phenotypic variations in cell [2]. It is well described in the literature that invasion, metastasis, and drug resistance are related to EMT [3] (Examples are Taxol and Adriamycin resistance in EMT activated tumors [4]). It is also well described in the literature that epigenetic status of the cancer cell lines (DNA methylation status) also correlates to the chemotherapy response and resistance (as shown in Gastric cancer cell lines response to platinum drugs) [5]. As our knowledge of cancer stem cells advances, we are able to identify the cancer stem cells quantitative measures of "stemness" that 
not only correlates with the EMT process, but also the epigenetic degree of aberrancies [6]. There is concomitant increase in the cancer "stem like" cells (CSC) that coexists with the degree of EMT [7]. This phenomenon is suggested to be under the regulation of epigenetics. Here we review the key targets in EMT, and further explore the therapeutic options that target these through epigenetic modification.

1) Transcription and growth factors/key pathways: Since EMT in cancer progression recaptures EMT in developmental process, several key inducers of EMT are transcription factors that repress the expression of E-cadherin, whose loss is a hallmark of EMT [3] [8]-[10]. Ras-MAPK, Hedgehog, and Phosphoinositol 3 kinase (PI3k) all have been suggested for triggering the EMT [11]-[13]. Transforming Growth Factor (TGF beta-1) is known to induce Insulin Like Growth Factor (IGF-1) and C-C Chemokine Ligand 2 (CCL2) in cancer, which promotes lymphatic spread of disease, and is also increased in hematological spread (i.e., in breast cancer with bone metastasis) [14]. TGF $\beta$ also can promote tumor invasion and evasion of immune surveillance [14] [15]. It is suggested that taxanes increase the production of TGF [16]. The same concern exists for Fibroblast Growth Factor-2 (FGF-2) and related induction of EMT [17]. Experiments with drugs that inhibit the FGF are still under research and development. In Gliomas, the involvement of bone marrow derived cells, their recruitment to the microenvironment, and participation in such "endocrine machinery" are critical for EMT induction, as well as tumor invasion and metastasis [18].

2) Hypoxia response elements and pathways: Hypoxia inducible factor-1 (HIF-1) and hypoxia response elements also play a role in triggering EMT activity. Notch1 and related SNAIL and SLUG have been described as key targets for EMT [9] [19]. SLUG is involved with the first stage, which contributes to the cell-cell adhesion, while the second phase requires other factors (induced Vimentin) [9] [20]. Several signaling pathways are involved with the hypoxia and Notch1 activation and result in EMT that can include the growth factors such as HGF, FGF, TGF beta-1 [9] [21]. SNAIL and SLUG both downregulate the p63, which is in charge of inhibiting the EMT [18] [22] [23]. SNAIL inhibits P53 related apoptosis [24]. P53 also activates the microRNAs which are inhibiting the EMT [25]. Wnt pathway also induces both SNAIL and SLUG, and upregulates the mesenchymal marker Vimentin [9]. SNAIL also confers resistance to Taxanes [16].

3) The role of epigenetic therapy: Epigenetic influence on almost all steps of cancer progression are shown in the literature, from carcinogenesis, to migration, invasion, and metastasis. This influence extends to the phenotypical and biological behavior of the disseminated circulatory tumor cells (CTCs), as well as free nucleic acid, or circulatory tumor DNA (ctDNA). It is well known that the tendency of circulatory tumor cells to spread and colonize in distant organs is an epigenetic phenomenon, as the mesenchymal CTCs have the ability to embolize and form colonies in distant organs. Chemotherapies, (shown with taxanes) are known for inducing EMT and phenotypical changes of CTCs in favor of metastasis [26]. It is time to explore epigenetic therapies that could potentially counteract such a pattern. A variety of genes are involved other than the hallmark pathways already described, whose epigenetic status can be assessed through the PCR technology. To name a few these genes: CDH1, DSP, KRT19, MST1R, OCLN, PPPDE2, RGS2, TSPAN13, Differentiation \& Development: MST1R, PTP4A1, SMAD4, Cell Growth \& Proliferation: GAB1, MST1R, SEH1L, SMAD4, Extracellular Matrix \& Cell Adhesion: CDH1, CTNNAL1, DSC2, EPCAM, NID2, Signal Transduction: GAB1, KRT19, MAP3K5, RGS2, SMAD4, TGIF1 and Cytoskeleton: CTNNAL1, KRT7, PLEK2 [9] [25].

As our understanding of the EMT expands, we are now able to differentiate three classes of biological behavior in EMT process. Therefore, we have noticed a spectrum of "EMT-ness" or degree of cell tendency to undergo EMT. Anything between full mesenchymal cell and full epithelial cell can fall in the middle, identified by quantitatively measured level of activity related to the bidirectional microRNAs (34 and 200) and the activity of ZEB and SNAIL, which transform the cell from partial EMT status to full EMT during cancer progression, induced by mechanisms described earlier [27] [28]. It is noticeable that microRNAs involved in this process are also non coding small RNAs that are involved in gene expression and part of the epigenetic science which has translated into diagnostic, as well as therapeutic tools in cancer [29]. The oncomiRNAs are RNAs which have been described as elevated during carcinogenesis and metastasis, as well as EMT, and the suppressor RNAs, which constitute the majority of microRNAs, can include: 16 in breast cancer, 21 in solid tumors such as lung, gastric, brain and ovaries, as well as hematological CA, 141 in prostate CA, and 198 lung CA [30]. These microRNAs are epigenetically controlled and may be modified, as their aberrancies are reversible.

Several tumorigenic properties driven or induced by EMT are modified by epigenetics. Epigenetic regulation encompasses three types of changes: DNA methylation, histone modifications, and microRNAs, each of which has been shown to play a key role in controlling epithelial-mesenchymal transition and cancer metastasis [5] [31] 
[32]. As we gain deeper understanding of epigenetic mechanisms controlling EMT processes and orchestrating all the metastatic steps, we broaden the therapeutic potentials of epigenetic drugs, such as DNA demethylating drugs and histone deacetylase/demethylase inhibitors [5] [31] [33]. EMT transcriptional regulation of E-cadherin is dependent on DNA methylation and histone decaetylation, which can act upon metastasis-related genes, restoring their expression and biological functions, orchestrating the heredity and reversibility of EMT [5]. It also has been shown in the literature that combination of histone deacetylase inhibitors along with demethylators can affect cancer cell differentiation in pancreatic cancer cell line model [5] [31] [33].

\section{Methods}

We have used a regimen of therapies that reverses the partial EMT status and induces MET, by regulating the key targets. Our approach may represent a promising therapeutic tool in advanced cancer. In this strategy we use treatments that inhibit the key regulators of EMT (such as SNAIL and SLUG) and the indirect pathways related to EMT (such as Wnt) [34]. The main component of the therapies are widely available and described in the literature, but the combination is discussed here for the first time, in a protocol called Multi-targeted epigenetic therapy (MTET), which addresses the key regulators involved with the EMT process. Of note, cytotoxic and cytostatic drugs are not used, as they induce the EMT.

1) Regulation of transcription and growth factors, by application of a combination of polyphenols that have been known to modify the pan-genomic and epi-genomic signature: This is the mainstay of the therapy, and is shown to be clinically relevant to improve survival in a series of cases, discussed here. The mechanism of action has been described in the literature, however the uniqueness of this protocol is related to the combination of several polyphenols that have synergism on the EMT targets. These polyphenols appear to affect the CSC invasion, migration, and self-renewal potency. The targets include caspase, survivin, BCL-2, LEF1/TCF responsive reporter, XIAP, and the number and self-renewal potency of CSC [35]-[40].

2) GnRH agonists: These are shown to affect the intratumoral hypoxia by inhibiting HIF-1. These therapies also inhibit the Ras-mediated MAPK, involved with EMT. Androgen inhibition, although useful in inhibiting the HIF-1 and growth factors such as VEGF, is suggested to induce EMT, however this is secondary to direct androgen blockade rather than GnRH agonist effect. There is also studies that show androgen receptor blockade as is the case for the drug (Firmagon) inhibits the production of VEGF, and related induction of EMT. VEGF-Amediated angiogenesis is the connecting mechanism between cancer cell stemness and tumor initiation [41].

3) Growth hormone blockade, by somatostatin analogues: These drugs are used to inhibit the IGF- 1 and TGF $\beta$ related cascade in EMT induction. It is described in the literature that blocking the IGF-1 mediation (through MUC1), inhibits EMT. IGF-1 is also a key target in induction of the PI3K mediated MAPK [42].

4) Quinolone based antimalaria drugs: These drugs inhibit the signal induction through growth factor receptor blockade. They also inhibit the Heat Shock Protein-90 (HSP-90) chaperones and PI3k/MAPK pathways, which are involved with EMT process [43].

5) Riluzole: An off label drug which has shown efficacy in preliminary findings on melanoma cancer cell lines, by inhibiting NMDA coupling with PI3k [44].

6) Targeted therapies, including EGFR blockade through small molecule inhibitors, such as Erlotinib: Some of the drugs in this category, known as EGFR tyrosine kinase inhibitors (TKIs) can inhibit the EMT, (shown in triple negative breast cancer cell lines in vitro) [45]. The EMT inhibition seems to be dose independent, and is not correlated with actual apoptosis effect. The other TKI used is Everolimus. This anti-mammalian target of Rapamycin, or mTOR inhibitor, also blocks EMT by inhibiting Wnt pathway. C-Met inhibitors, such as crizotonib, are also studied and have shown promising results in inhibiting CSC, to the point that some believe that EGFR blockade resistance can be reversed by adding c-Met inhibitors [46]. C-Met is an interesting target, as it is shown that it is crucial for the CSC self-renewal, and also an equivalent biomarker already described as CD 44+alpha2, beta1, or CD133+ [47]. Some targeted therapies induce the EMT, shown by activation of Wnt pathways after using the new MEK inhibitor, Selumetenib [48]. Metformin has been studied as a therapeutic in cancer cell lines, and works by inhibition of mTOR as well as positive effect on CSC, but must be used cautiously, as it can induce the feedback loops that trigger the PI3k [49].

7) Celecoxib: there is controversy regarding the use of celecoxib, as it appears in some studies to induce EMT, although it was previous thought to inhibit EMT, and synergizes with EGFR blockade [50].

Accordingly application of the above protocol in one hundred cases of advanced and refractory solid tumors 
resulted in independent anti-EMT response identified by disseminated circulatory tumor cells, translated to improved progression free, or overall survival. We followed the plasma VEGF measurements, as an indirect biomarker for intratumoral hypoxia, the IGF-1 and TGF-beta 1, as growth factor markers and epithelial circulatory tumor cells (EpCAM), altogether, a meaningful companion diagnostic tool to translate to clinical outcome, and predict overall survival. We conclude that this sample, although small, presents considerable effect size and can impact the current practice of oncology by providing better prognostic and therapeutic tools targeting CTC and cancer stem cell "stemness" in refractory and advanced disease.

\section{Results}

The following results were obtained during or after completing the course of therapy:

1) Response in tumor markers, suggesting clinical response: Of 100 patients, 73 patients initially had elevated tumor markers, and 57 of those patients (78 percent) had decreased tumor markers after the course of treatment. Three patients had stable numbers, and four patients had continuously increased markers. Nine patients had fluctuating results (decreased initially, and then increased).

2) Shrinkage of tumor in radiographic/imaging studies (PET and CT) with partial/mixed response and decreased metabolic activity: 58 patients had imaging reports to follow for their condition. The other 42 had no imaging or it was not applicable or relevant. Of 58 patients, 36 patients (62 percent) had positive response in their scans after the treatments. In 17 patients the results were mixed or improved initially, or the tumor was stabilized (total response of 91 percent). Five cases (8 percent) continued to progress.

3) Decrease in IGF-1: Of 100 patients, 20 had increased IGF-1. (The remaining had normal IGF-1). 18 of 20 patients (80 percent) responded by decreased levels after the treatment, (suggested to correlate with survival in the literature). In two patients IGF-1 increased.

4) Decrease in VEGF/FGF-2/IL-8: 37 patients had increased vascular endothelial growth factor (VEGF), correlating with higher risk of metastasis. 34 patients had decreased VEGF after the treatments (92 percent). Three patients progressed despite therapy (8 percent).

5) Circulatory tumor cells: 20 patients had increased CTC. 16 patients had positive circulatory tumor cells response by lowered CTC, which correlates with both disease free and overall survival post treatment. One patient had increased CTCs, and three had mixed responses (initially improved, and then progressed).

Since patients with cancer may have significant stratifying confounders in selecting their control group, we used each patients' pre-interventional status as the control arm. Patients' other stratifying confounders did not change during the study.

\section{Discussion}

Many of these patients had exhausted standard of care treatments, had stage IV disease, and had been offered palliative chemotherapy. The high rate of response in markers such as VEGF, PET imaging, and CTC has been show in the literature to correlate with survival. None of the patients treated with MTET experienced decreased quality of life, measured by ECOG performance status.

\section{Conclusion}

Our preliminary findings suggest that the implementation of anti-EMT therapies through MTET protocol is a promising therapeutic approach that warrants further research and investigation.

\section{References}

[1] Vija, L., Farge, D., Gautier, J., Vexiau, P. and Dumitrache, C. (2008) Mesenchymal Stem Cells: Stem Cell Therapy Perspectives for Type 1 Diabetes. Diabetes \& Metabolism, 2015, 85-93.

[2] Nakaya, Y. and Sheng, G.J. (2013) EMT in Developmental Morphogenesis. Cancer Letters, 341, 9-15. http://dx.doi.org/10.1016/j.canlet.2013.02.037

[3] Kalluri, R. and Weinberg, R.A. (2009) The Basics of Epithelial-Mesenchymal Transition. Journal of Clinical Investigation, 119, 1420-1428. http://dx.doi.org/10.1172/JCI39104

[4] Wang, Z., Li, Y., Ahmad, A., Azmi, A., Kong, D. and Banerjee, S. (2010) Targeting miRNAs Involved in Cancer Stem 
Cell and EMT Regulation: An Emerging Concept in Overcoming Drug Resistance. Drug Resistance Updates, 13, 109118. http://dx.doi.org/10.1016/j.drup.2010.07.001

[5] Ivanova, T., Zouridis, H., Wu, Y., Cheng, L., Tan, I. and Gopalakrishnan, V. (2012) Integrated Epigenomics Identifies BMP4 as a Modulator of Cisplatin Sensitivity in Gastric Cancer. Gut, 62, 22-33.

[6] Neureiter, D. (2012) Epigenetic Control of Epithelial-Mesenchymal-Transition in Human Cancer (Review). Molecular and Clinical Oncology, 2, 3-11. http://dx.doi.org/10.3892/mco.2012.28

[7] Liu, X. and Fan, D. (2015) The Epithelial-Mesenchymal Transition and Cancer Stem Cells: Functional and Mechanistic Links. Current Pharmaceutical Design, 21, 1279-1291. http://dx.doi.org/10.2174/1381612821666141211115611

[8] Peinado, H., Olmeda, D. and Cano, A. (2007) Snail, Zeb and bHLH Factors in Tumour Progression: An Alliance against the Epithelial Phenotype? Nature Reviews Cancer Nat Rev Cancer, 7, 415-428.

[9] Yang, J. and Weinberg, R. (2008) Epithelial-Mesenchymal Transition: At the Crossroads of Development and Tumor Metastasis. Developmental Cell, 14, 818-829. http://dx.doi.org/10.1016/j.devcel.2008.05.009

[10] Kurrey, N., Jalgaonkar, S., Joglekar, A., Ghanate, A., Chaskar, P., Doiphode, R. and Bapat, S. (2009) Snail and Slug Mediate Radioresistance and Chemoresistance by Antagonizing p53-Mediated Apoptosis and Acquiring a Stem-Like Phenotype in Ovarian Cancer Cells. Stem Cells, 27, 2059-2068. http://dx.doi.org/10.1002/stem.154

[11] Vlahopoulos, S., Logotheti, S., Mikas, D., Giarika, A., Gorgoulis, V., et al. (2008) The Role of ATF-2 in Oncogenesis. BioEssays, 30, 314-327. http://dx.doi.org/10.1002/bies.20734

[12] Huber, M., Beug, H. and Wirth, T. (2004) Epithelial-Mesenchymal Transition: NF- $\kappa$ B Takes Center Stage. Cell Cycle, 3, 1477-1480. http://dx.doi.org/10.4161/cc.3.12.1280

[13] Katoh, Y. and Katoh, M. (2008) Hedgehog Signaling, Epithelial-to-Mesenchymal Transition and miRNA (Review). International Journal of Molecular Medicine, 22, 271-275.

[14] Fuxe, J. and Karlsson, M. (2012) TGF- $\beta$-Induced Epithelial-Mesenchymal Transition: A Link between Cancer and Inflammation. Seminars in Cancer Biology, 22, 455-461. http://dx.doi.org/10.1016/j.semcancer.2012.05.004

[15] Zhang, J.Y., Tian, X.J., Zhang, H., Teng, Y., Li, R.Y., Bai, F., Elankumaran, S. and Xing, J.H. (2014) TGF- $\beta$-Induced Epithelial-to-Mesenchymal Transition Proceeds through Stepwise Activation of Multiple Feedback Loops. Science Signaling, 7, ra91. http://dx.doi.org/10.1126/scisignal.2005304

[16] Cui, S.Y., Wang, R. and Chen, L.B. (2013) MicroRNAs: Key Players of Taxane Resistance and Their Therapeutic Potential in Human Cancers. Journal of Cellular and Molecular Medicine, 17, 1207-1217. http://dx.doi.org/10.1111/jcmm.12131

[17] Lee, J.G. and Kay, E.P. (2006) FGF-2-Mediated Signal Transduction during Endothelial Mesenchymal Transformation in Corneal Endothelial Cells. Experimental Eye Research, 83, 1309-1316. http://dx.doi.org/10.1016/j.exer.2006.04.007

[18] Herfs, M., Hubert, P., Suarez-Carmona, M., Reschner, A., Saussez, S., Berx, G., Savagner, P., Boniver, J. and Delvenne, P. (2010) Regulation of p63 Isoforms by Snail and Slug Transcription Factors in Human Squamous Cell Carcinoma. The American Journal of Pathology, 176, 1941-1949. http://dx.doi.org/10.2353/ajpath.2010.090804

[19] Chakrabarti, R., Hwang, J., Blanco, M., Wei, Y., Lukačišin, M., et al. (2012) Elf5 Inhibits the Epithelial-Mesenchymal Transition in Mammary Gland Development and Breast Cancer Metastasis by Transcriptionally Repressing Snail2. Nature Cell Biology, 14, 1212-1222. http://dx.doi.org/10.1038/ncb2607

[20] Savagner, P. (2010) The Epithelial-Mesenchymal Transition (EMT) Phenomenon. Annals of Oncology, 21, Vii89Vii92. http://dx.doi.org/10.1093/annonc/mdq292

[21] Savagner, P., Yamada, K.M. and Thiery, J.P. (1997) The Zinc-Finger Protein Slug Causes Desmosome Dissociation, an Initial and Necessary Step for Growth Factor-Induced Epithelial-Mesenchymal Transition. The Journal of Cell Biology, 137, 1403-1419. http://dx.doi.org/10.1083/jcb.137.6.1403

[22] Lindsay, J., Mcdade, S., Pickard, A., Mccloskey, K. and Mccance, D. (2010) Role of $\Delta$ Np63 $\gamma$ in Epithelial to Mesenchymal Transition. Journal of Biological Chemistry, 286, 3915-3924. http://dx.doi.org/10.1074/jbc.M110.162511

[23] Boldrup, L., Coates, P., Gu, X. and Nylander, K. (2007) $\Delta$ Np63 Isoforms Regulate CD44 and Keratins 4, 6, 14 and 19 in Squamous Cell Carcinoma of Head and Neck. The Journal of Pathology, 213, 384-391. http://dx.doi.org/10.1002/path.2237

[24] Firdous, A., Sharmila, G., Balakrishnan, S., Rajasingh, P., Suganya, S., et al. (2014) Quercetin, a Natural Dietary Flavonoid, Acts as a Chemopreventive Agent against Prostate Cancer in an in Vivo Model by Inhibiting the EGFR Signaling Pathway. Food \& Function, 5, 2632-2645. http://dx.doi.org/10.1039/C4FO00255E

[25] Kang, Y.B., He, W., Tulley, S., Gupta, G.P., Serganova, I., Chen, C.-R., Manova-Todorova, K., Blasberg, R., Gerald, W.L. and Massagué, J. (2005) Breast Cancer Bone Metastasis Mediated by the Smad Tumor Suppressor Pathway. Proceedings of the National Academy of Sciences of the United States of America, 102, 13909-13914. http://dx.doi.org/10.1073/pnas.0506517102 
[26] Pachmann, K., Camara, O., Kavallaris, A., Krauspe, S., Malarski, N., Gajda, M., et al. (2008) Monitoring the Response of Circulating Epithelial Tumor Cells to Adjuvant Chemotherapy in Breast Cancer Allows Detection of Patients at Risk of Early Relapse. Journal of Clinical Oncology, 26, 1208-1215. http://dx.doi.org/10.1200/JCO.2007.13.6523

[27] Chang, C.-J., Chao, C.-H., Xia, W.Y., Yang, J.-Y., Xiong, Y., Li, C.-W., Yu, W.-H., Rehman, S.K., Hsu, J.L., Lee, H.-H., Liu, M., Chen, C.-T., Yu, D.H. and Hung, M.-C. (2011) P53 Regulates Epithelial-Mesenchymal Transition and Stem Cell Properties through Modulating miRNAs. Nature Cell Biology, 13, 317-323. http://dx.doi.org/10.1038/ncb2401

[28] Lu, M., Jolly, M., Levine, H., Onuchic, J. and Ben-Jacob, E. (2013) MicroRNA-Based Regulation of Epithelial-Hybrid-Mesenchymal Fate Determination. Proceedings of the National Academy of Sciences of the United States of America, 110, 18144-18149. http://dx.doi.org/10.1073/pnas.1318192110

[29] Craene, B. and Berx, G. (2013) Regulatory Networks Defining EMT during Cancer Initiation and Progression. Nature Reviews Cancer, 13, 97-110. http://dx.doi.org/10.1038/nrc3447

[30] Grange, C., Collino, F., Tapparo, M. and Camussi, G. (2014) Oncogenic Micro-RNAs and Renal Cell Carcinoma. Frontiers in Oncology, 4, 49. http://dx.doi.org/10.3389/fonc.2014.00049

[31] Huangyang, P.W. and Shang, Y.F. (2013) Epigenetic Regulation of Epithelial to Mesenchymal Transition. Current Cancer Drug Targets, 13, 973-985. http://dx.doi.org/10.2174/15680096113136660103

[32] Tian, X.J., Zhang, H. and Xing, J.H. (2013) Coupled Reversible and Irreversible Bistable Switches Underlying TGF $\beta$-Induced Epithelial to Mesenchymal Transition. Biophysical Journal, 105, 1079-1089. http://dx.doi.org/10.1016/j.bpj.2013.07.011

[33] Wang, Y. and Shang, Y.F. (2013) Epigenetic Control of Epithelial-to-Mesenchymal Transition and Cancer Metastasis. Experimental Cell Research, 319, 160-169. http://dx.doi.org/10.1016/j.yexcr.2012.07.019

[34] Micalizzi, D., Farabaugh, S.M. and Ford, H.L. (2010) Epithelial-Mesenchymal Transition in Cancer: Parallels between Normal Development and Tumor Progression. Journal of Mammary Gland Biology and Neoplasia, 15, 117-134. http://dx.doi.org/10.1007/s10911-010-9178-9

[35] Chang, Y.C., Chen, P.N., Chu, S.C., Lin, C.Y., Kuo, W.H., et al. (2012) Black Tea Polyphenols Reverse Epithelial-to-Mesenchymal Transition and Suppress Cancer Invasion and Proteases in Human Oral Cancer Cells. Journal of Agricultural and Food Chemistry, 60, 8395-8403. http://dx.doi.org/10.1021/jf302223g

[36] Lin, C.H., Shen, Y.A., Hung, P.H., Yu, Y.B. and Chen, Y.J. (2012) Epigallocathechin Gallate, Polyphenol Present in Green Tea, Inhibits Stem-Like Characteristics and Epithelial-Mesenchymal Transition in Nasopharyngeal Cancer Cell Lines. BMC Complementary and Alternative Medicine, 12, 201. http://dx.doi.org/10.1186/1472-6882-12-201

[37] Belguise, K., Guo, S., Yang, S., Rogers, A., Seldin, D., et al. (2007) Green Tea Polyphenols Reverse Cooperation between c-Rel and CK2 That Induces the Aryl Hydrocarbon Receptor, Slug, and an Invasive Phenotype. Cancer Research, 67, 11742-11750. http://dx.doi.org/10.1158/0008-5472.CAN-07-2730

[38] Tang, S.N., Singh, C., Nall, D., Meeker, D., Shankar, S., et al. (2010) The Dietary Bioflavonoid Quercetin Synergizes with Epigallocathechin Gallate (EGCG) to Inhibit Prostate Cancer Stem Cell Characteristics, Invasion, Migration and Epithelial-Mesenchymal Transition. Journal of Molecular Signaling, 5, 14. http://dx.doi.org/10.1186/1750-2187-5-14

[39] Lu, Q., Ji, X.J., Zhou, Y.X., Yao, X.Q., Liu, Y.Q., et al. (2015) Quercetin Inhibits the mTORC1/p70S6K Signaling-Mediated Renal Tubular Epithelial-Mesenchymal Transition and Renal Fibrosis in Diabetic Nephropathy. Pharmacological Research, 99, 237-247. http://dx.doi.org/10.1016/j.phrs.2015.06.006

[40] Bhat, F., Sharmila, G., Balakrishnan, S., Arunkumar, R., Elumalai, P., et al. (2014) Quercetin Reverses EGF-Induced Epithelial to Mesenchymal Transition and Invasiveness in Prostate Cancer (PC-3) Cell Line via EGFR/PI3K/Akt Pathway. The Journal of Nutritional Biochemistry, 25, 1132-1139. http://dx.doi.org/10.1016/j.jnutbio.2014.06.008

[41] Fantozzi, A., Gruber, D., Pisarsky, L., Heck, C., Kunita, A., Yilmaz, M., Meyer-Schaller, N., Cornille, K., Hopfer, U. Bentires-Alj, M. and Christofori, G. (2014) VEGF-Mediated Angiogenesis Links EMT-Induced Cancer Stemness to Tumor Initiation. Cancer Research, 74, 1566-1575. http://dx.doi.org/10.1158/0008-5472.CAN-13-1641

[42] Liao, G., Wang, M., Ou, Y. and Zhao, Y. (2013) IGF-1-Induced Epithelial-Mesenchymal Transition in MCF-7 Cells Is Mediated by MUC1. Cellular Signalling, 26, 2131-2137. http://dx.doi.org/10.1016/j.cellsig.2014.06.004

[43] Kumar, R., Musiyenko, A. and Barik, S. (2003) The Heat Shock Protein 90 of Plasmodium Falciparum and Antimalarial Activity of Its Inhibitor, Geldanamycin. Malaria Journal, 2, 30. http://dx.doi.org/10.1186/1475-2875-2-30

[44] Bukhsh, M., Speyer, C., Hachem, A., Nassar, M., Assi, A. and Gorski, D. (2014) Abstract 4608: Exploring Anti-Oncogenic Properties of Riluzole in Breast Cancer. Cancer Research, 74, 4608. http://dx.doi.org/10.1158/1538-7445.AM2014-4608

[45] Corkery, B., Crown, J., Clynes, M. and O’donovan, N. (2009) Epidermal Growth Factor Receptor as a Potential Therapeutic Target in Triple-Negative Breast Cancer. Annals of Oncology, 20, 862-867. 
http://dx.doi.org/10.1093/annonc/mdn710

[46] Avan, A., Quint, K., Nicolini, F., Funel, N., Frampton, A., Maftouh, M., Pelliccioni, S., Schuurhuis, G.J., Peters, G.J. and Giovannetti, E. (2013) Enhancement of the Antiproliferative Activity of Gemcitabine by Modulation of c-Met Pathway in Pancreatic Cancer. Current Pharmaceutical Design, 19, 940-950. http://dx.doi.org/10.2174/138161213804547312

[47] Chen, W., Wang, G.M., Liu, Y.J. and Qian, R.Z. (2007) Cancer Stem-Like Cells in Human Prostate Carcinoma Cells DU145: The Seeds of the Cell Line? Cancer Biology \& Therapy, 6, 763-768. http://dx.doi.org/10.4161/cbt.6.5.3996

[48] Grasso, S., Tristante, E., Saceda, M., Carbonell, P., Mayor-López, L., Carballo-Santana, M., Carrasco-García, E., Rocamora-Reverte, L., García-Morales, P., Carballo, F., Ferragut, J.A., Martínez-Lacaci, I. (2014) Resistance to Selumetinib (AZD6244) in Colorectal Cancer Cell Lines Is Mediated by p70S6K and RPS6 Activation. Neoplasia, 16, 845860. http://dx.doi.org/10.1016/j.neo.2014.08.011

[49] Honjo, S., Ajani, J., Scott, A., Chen, Q. and Skinner, H. (2014) Metformin Sensitizes Chemotherapy by Targeting Cancer Stem Cells and the mTOR Pathway in Esophageal Cancer. International Journal of Oncology, 45, 567-574. http://dx.doi.org/10.3892/ijo.2014.2450

[50] Bocca, C., Bozzo, F., Cannito, S., Parola, M. and Miglietta, A. (2011) Celecoxib Inactivates Epithelial-Mesenchymal Transition Stimulated by Hypoxia and/or Epidermal Growth Factor in Colon Cancer Cells. Molecular Carcinogenesis, 51, 783-795. http://dx.doi.org/10.1002/mc.20846 